\title{
PASSENGER TRANSFER EFFICIENCY OPTIMIZATION MODELLING RESEARCH WITH SIMULATIONS
}

\author{
Feng, X. ; Wang, X. \& Zhang, H. \\ MOE Key Laboratory for Urban Transportation Complex Systems Theory \& Technology, \\ Beijing Jiaotong University, No. 3 Shangyuancun, Haidian District, Beijing 100044, P. R. China \\ E-Mail: xsfeng@bjtu.edu.cn ( ${ }^{*}$ Corresponding author)
}

\begin{abstract}
An integrated optimization model is newly developed in this research to improve passenger transfer efficiency of an urban rail station by minimizing average waiting time of transfers in this station with rationalizing the arrival and departure time of trains in view of transfer passenger flows and their feeder services. Corresponding algorithm is also designed to search for the optimal solution to the objective function of the proposed model. A representative urban rail transfer station in Beijing is studied as example to validate the effect of transfer optimization with the new model. Comparative simulations confirm that the proposed model is able to effectively reduce the waiting time of each transfer in a station. This is significant for systematically improving the operation safety of an urban rail transfer station with faster evacuations of transfer passengers as well as substantially saving travel time of passengers making transfers in this station.

(Received, processed and accepted by the Chinese Representative Office.)
\end{abstract}

Key Words: Passenger Transfer Waiting Time, Urban Rail Transport Station, Train Arrival and Departure Time, Optimization Modelling, Simulation Analysis

\section{INTRODUCTION}

Though automobile industry is very important in national economy [1], resource and environment problems slow down the automobile transport development in China. Therewith, Chinese rail transport develops very fast in recent years as a both sustainable and efficient transport mode especially for the passenger transports within not only regional but also urban areas $[2,3]$. For the purpose of providing efficient transport services, the passenger transfer efficiency of an urban rail transfer station should be improved as much as possible in particular for its peak time. In general, the passenger transfer efficiency can be increased by improving transfer facilities or rationalizing train operation diagrams [4]. In view of the big money and time costs for the alteration or reconstruction of a facility which is always huge and immovable in a rail station, optimizing train operation plans is more feasibly effective to improve the transfer efficiency of a station. Such an optimization issue is ordinarily solved by taking the arrival and departure time of trains as decision variables to make a mixed integer linear programming modelling study for the minimal waiting time of a passenger in a transfer [5]. In this manner, the operation diagrams for the trains running on different rail lines on the same rail network are also able to get synchronization optimizations [5]. Moreover, many other factors such as transfer times of a passenger's trip can be optimized synchronously as well [6]. Furthermore, shortening transfer waiting time also improves the operation safety of an especially underground station with more enclosed and very limited internal space by decreasing the duration of its stranding a large number of transfer passengers inside.

In order to explore rational and efficient approaches for such transfer efficiency optimizations, many efforts have been made continually. For instance, an optimization method able to effectively set buffer time on train diagrams is proposed by Vansteenwege and Oudheusden [7] to ensure the continuity of transfers in case of train delays. Heuristic methods [8] are utilized in the work of Wong et al. [9] to ameliorate the train operation diagrams of an 
urban rail network according to different transfer characteristics of passengers for saving transfer waiting time. Liebchen [10] takes advantage of Graph Theory [11] to determine the arrival and departure time of trains for the minimal transfer waiting time of passengers. Khani and Shafahi [12] develop an optimization model based on headways and departure time of trains to reduce the waiting time of passengers in their transfers. In addition, because transfer waiting time is also mainly affected by the capacities and headways of the connecting and feeder services [13], a transfer optimization model with the constraints of feeder services is developed by Ying et al. [14]. The existing studies have put forward various valuable means and have got many precious findings. However, the constraints of feeder services have not been adequately analysed from a systematic perspective for the integrated optimization of different transfers in a rail station. As a result, this research newly develops an integrated optimization model focusing on rationalizing the arrival and departure time of trains to minimize the average waiting time of different transfers in consideration of the capacities of feeder services and the volumes of transfer passengers for all the transfers in an urban rail station. In this way, the transfer operation efficiency of the station is able to get an overall improvement.

The contents in the latter parts of this paper are organized as follows. Section 2 introduces the new integrated optimization model. Then, the algorithm utilized to solve the optimization problem interpreted by the new model is provided in Section 3. Next, typical combinations of different transfer passenger flows in various time periods of a commuting day is studied in Section 4 for a representative metro transfer station in Beijing to improve its average transfer efficiency with applying the newly developed optimization model and the efficiency improvement effect is proved with comparative simulations. Finally, Section 5 makes the conclusions and points out some future research issues.

\section{MODEL ESTABLISHMENT}

A rail transfer station is often able to simultaneously provide inside transfers in different directions. For instance, as shown in Fig. 1, Xierqi Station represented here by $\mathrm{X}$ is both an intermediate station of Line No. 13 and a terminal station of Changping Line on the metro network in Beijing. It can offer 4 transfers, i.e. Transfer-1 (from A to B), Transfer-2 (from A to C), Transfer-3 (from B to A) and Transfer-4 (from C to A).

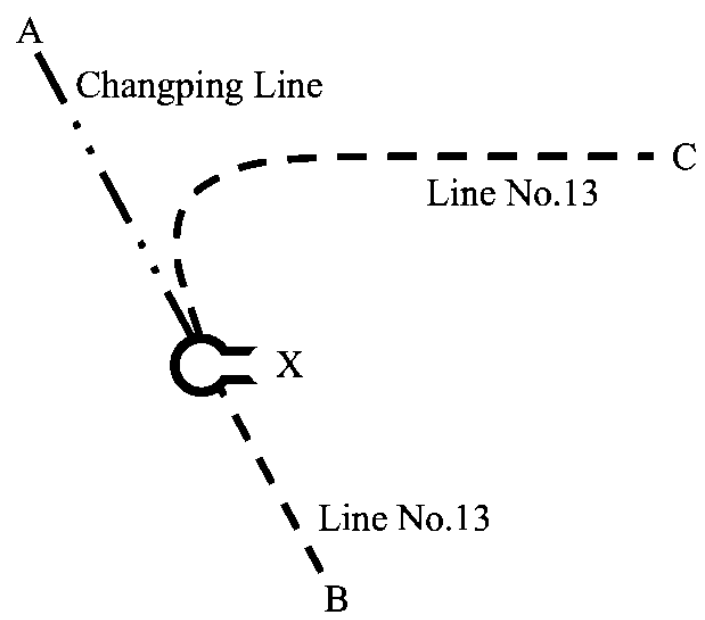

Figure 1: Xierqi Station in Beijing.

If the transfer facilities in a rail station are not improved, the time used for a transfer passenger's getting off a train, walking to the boarding site and boarding the connecting train 
in this station is relatively fixed. In this case, the transfer operation efficiency of a rail station in a certain time may be well evaluated by the average transfer waiting time of a passenger making a transfer in this station. Minimizing the average transfer waiting time is also one of the goals of the urban rail transport operation management. Therefore, in this research, the objective function of the proposed optimization model for the minimal average waiting time of a passenger making a transfer in a certain urban rail station is explained by:

$$
\operatorname{Min} T=\operatorname{Min}\left(\sum_{k=1}^{l} P_{k} \sum_{i=1}^{n} \sum_{j=1}^{m} R_{i j} T_{i j}\right)
$$

where $T$ is the average transfer waiting time of a passenger making a transfer in a station, unit: seconds (s); $P_{k}$ represents the volume proportion of the passengers who make transfer $k$ in the total volume of all the transfer passengers in a station, unit: $\% ; R_{i j}$ indicates the probability of boarding train $j$ for a passenger who gets off train $i$ in transfer $k$, unit: \%; $T_{i j}$ donates the average waiting time of a passenger who transfers from train $i$ to train $j$ after arriving at the boarding site and before the arrival of train $j$, unit: $\mathrm{s} ; l$ means the number of all the transfers in the station, $n$ is the number of all the trains which arrive at the station in a certain time period and are utilizable for transfer $k$, and $m$ represents the number of all the trains which depart from the station in a certain time period and are utilizable for transfer $k$.

The total transfer passenger flow in a station consists of the transfer passenger flows in different directions, as interpreted by:

$$
\sum_{k=1}^{l} P_{k}=1,\left(0<P_{k}<1\right)
$$

The transfer waiting time of a passenger in a station is much determined with the arrival and departure time of the connecting train if there are no improvements to the transfer facilities, as shown in:

$$
T_{i j}=t_{j}^{\text {Dep }}-t_{i}^{A r r}-\left(\Delta t_{i}^{O f f}+\Delta t_{i j}^{\text {Walk }}+\Delta t_{j}^{O n}\right)
$$

where $t_{j}^{\text {Dep }}$ donates the departure time of train $j, t_{i}^{\text {Arr }}$ represents the arrival time of train $i$, $\Delta t_{i}^{\text {Off }}$ is the average time consumed for a passenger's getting off train $i$, unit: $\mathrm{s} ; \Delta t_{i j}^{\text {Walk }}$ indicates the average time spent for a passenger's walking from the site of getting off train $i$ to the place to board train $j$, unit: s; and $\Delta t_{j}^{O n}$ means the average time used for a passenger' boarding train $j$, unit: s.

In view of the capacities of connecting trains and the volume of transfer passengers in a time period of a day, a passenger flow from an arrived train for the same transfer is usually split into a certain number of connecting trains with right adequate connecting time rather than all the trains with theoretically enough connecting time, as explained by:

$$
R_{i j} \begin{cases}=0, & \text { if }\left(T_{i j}<0\right) \text { or }\left(T_{i j}>C_{D e p} \times H_{D e p}^{M a x}\right) \operatorname{or}\left(\left(0 \leq T_{i j} \leq C_{D e p} \times H_{D e p}^{M a x}\right) \&\left(T_{i\left(j-C_{D e p}\right)} \geq 0\right)\right) \\ >0 \&<1, & \text { if }\left(0 \leq T_{i j} \leq C_{D e p} \times H_{D e p}^{M a x}\right) \&\left(T_{i\left(j-C_{D e p}\right)}<0\right) \&\left(C_{D e p}>1\right)\end{cases}
$$

where $C_{D e p}$ represents the number of the trains boarded by all the transfer passengers who get off one arrived train for transfer $k$ in a certain time period, and $H_{D e p}^{M a x}$ is the maximum headway of the trains which transport the transfer passengers right after their transfers for transfer $k$ in a certain time period, unit: $\mathrm{s}$. The number of the trains boarded by all the transfer passengers who get off one arrived train and make the same transfer and the probability of boarding such a connecting train for a transfer passenger are both generally unchanged for a 
given time in the daily operation of a station. By site research, the number of such necessary trains and the probabilities of boarding each of these trains, i.e. in other words the volume percentages of the passengers who get off the same train and board different connecting trains for the same transfer in the total volume of all these passengers, can be obtained normally.

Besides the afore-interpreted factors, the proposed model in this research also ought to follow the constraints of the minimum and maximum headways and tracking intervals of trains from the operation perspective of rail transport $[15,16]$ for the optimization of transfers, as explained by:

$$
\begin{gathered}
H_{A r r}^{M i n} \leq t_{i}^{A r r}-t_{i-1}^{A r r} \leq H_{A r r}^{M a x} \\
h_{A r r}^{M i n} \leq t_{i}^{A r r}-t_{i-1}^{D e p} \leq h_{A r r}^{M a x} \\
H_{D e p}^{M i n} \leq t_{j}^{D e p}-t_{j-1}^{D e p} \leq H_{D e p}^{M a x} \\
h_{D e p}^{M i n} \leq t_{j}^{A r r}-t_{j-1}^{D e p} \leq h_{D e p}^{M a x}
\end{gathered}
$$

where $H_{A r r}^{\text {Min }}$ means the minimum headway of the trains which transport the transfer passengers straight before their transfers for transfer $k$ in a certain time period, unit: s; $H_{A r r}^{M a x}$ represents the maximum headway of the trains which transport the transfer passengers right before their transfers for transfer $k$ in a certain time period, unit: $\mathrm{s} ; h_{A r r}^{\mathrm{Min}}$ is the minimum tracking interval of the trains which transport the transfer passengers straight before their transfers for transfer $k$ in a certain time period, unit: $\mathrm{s}$; $h_{A r r}^{M a x}$ indicates the maximum tracking interval of the trains which transport the transfer passengers right before their transfers for transfer $k$ in a certain time period, unit: s; $H_{D e p}^{M i n}$ represents the minimum headway of the trains which transport the transfer passengers right after their transfers for transfer $k$ in a certain time period, unit: $\mathrm{s} ; h_{\text {Dep }}^{\text {Min }}$ donates the minimum tracking interval of the trains which transport the transfer passengers right after their transfers for transfer $k$ in a certain time period, unit: s; and $h_{D e p}^{M a x}$ is the maximum tracking interval of the trains which transport the transfer passengers right after their transfers for transfer $k$ in a certain time period, unit: s.

\section{ALGORITHM DESIGN}

The optimization algorithm designed here to search for the optimum solution to the issue explained by the afore-interpreted newly developed model starts its process from the transfer with the biggest transfer passenger flow in comparison to the ones of the other transfers in a station for a certain time period of an operation day. This is able to effectively avoid local optimal solutions. The complete procedure of the proposed algorithm is illustrated in Fig. 2 and its calculation amount depends on the required accuracy of its outputs. Therefore, the optimization process is performed according to actual needs including rational requirements to its output accuracy in the application of the algorithm. The details in each step of the whole optimization procedure are as follows:

Step 1: Calculate $T$ of all the transfers within a certain time of a day in a station on the basis of the unoptimized arrival and departure time of trains and store $T$ as $T^{\prime}$;

Step 2: Find the transfer which has the biggest transfer passenger flow in this station for the target time period and record this transfer;

Step 3: Decide connecting train(s) of train $i(=1,2, \ldots, n)$ in this transfer according to (4);

Step 4: Adjust the departure time of the connecting train(s) for every possibility within the constraints of (8) and (9) and compute new $T$; if new $T$ is smaller than $T^{\prime}$, update $T^{\prime}$, the arrival time of train $i$ and the departure time of the connecting train(s); 
Step 5: Change the arrival time of train $i$ by following the requirements of (6) and (7) and go back to Step 3 until all the possible arrival time of train $i$ has been analysed;

Step 6: Determine the next transfer with the biggest transfer passenger flow in the unrecorded transfer(s), record it and go back to Step 3 until all the transfers have been investigated.

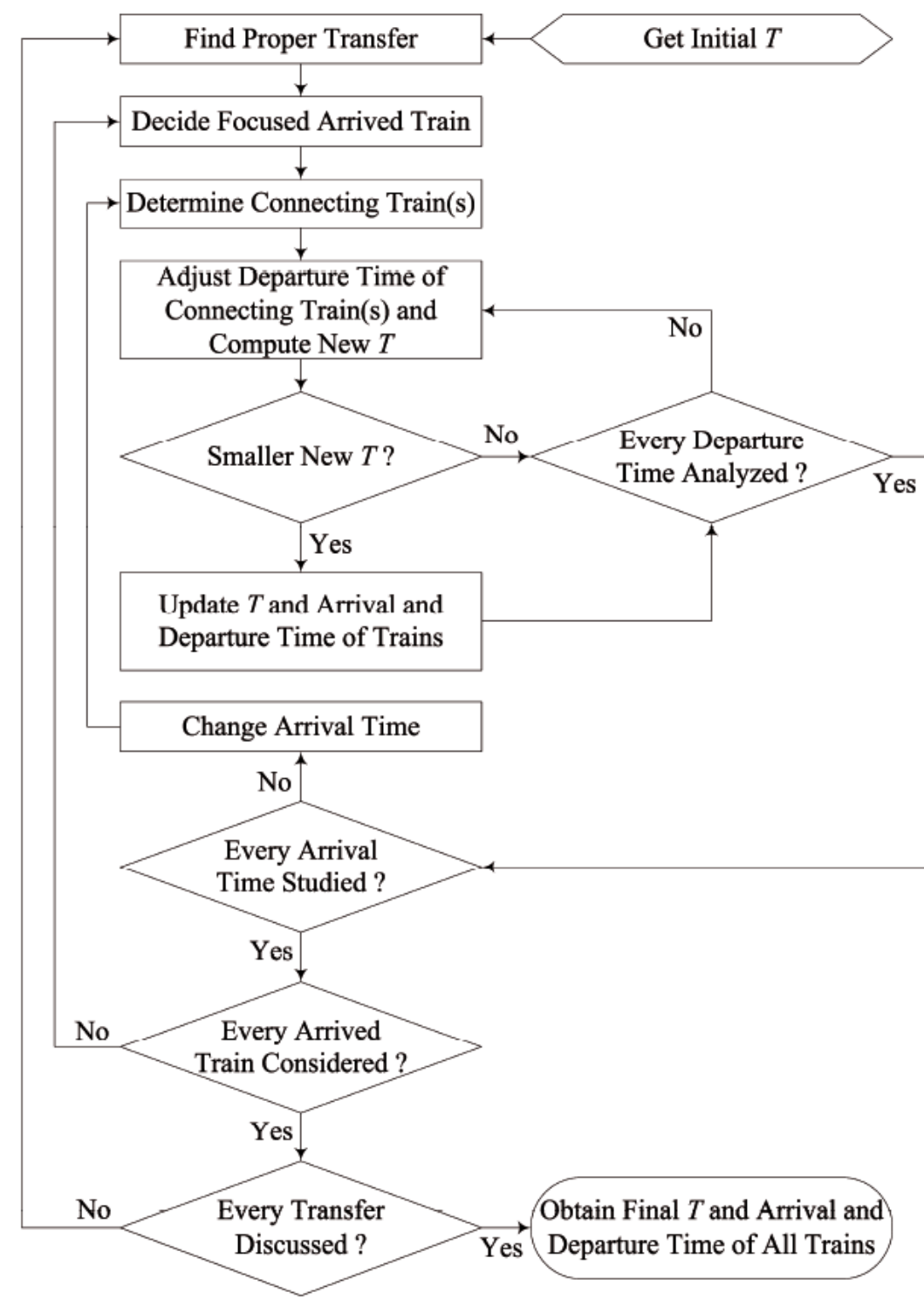

Figure 2: Optimization algorithm flowchart.

\section{OPTIMIZATION SIMULATION}

As afore-illustrated in Fig. 1, Xierqi Station (indicated as X) offers 4 transfers, i.e. Transfer-1 (from A to B), Transfer-2 (from A to C), Transfer-3 (from B to A) and Transfer-4 (from C to A). People making their transfers in this station often have to spend much time in waiting for connecting trains in various time periods of a day. In this research, the arrival and departure time of trains are adjusted with the proposed model to respectively minimize the transfer waiting time of a passenger in morning and evening rush hours and representative off-peak period of $\mathrm{X}$ in a commuting day. Parameters of the proposed optimization model for morning and evening peak periods and non-peak hour of $\mathrm{X}$ are correspondingly presented in Table I, Table II and Table III according to field survey. 
In these three tables, $P_{1}, P_{2}, P_{3}$ and $P_{4}$ are the proportions of the passengers making Transfer-1, Transfer-2, Transfer-3 and Transfer-4. $\Delta t_{A B}^{\text {Walk }}, \Delta t_{A C}^{\text {Walk }}, \Delta t_{B A}^{\text {Walk }}$ and $\Delta t_{C A}^{\text {Walk }}$ mean the time for walking from the sites of getting off trains from $\mathrm{A}, \mathrm{B}$ and $\mathrm{C}$ to the places to board the connecting trains to $\mathrm{B}, \mathrm{C}$ and $\mathrm{A} . \Delta t_{A}^{O f f}, \Delta t_{B}^{\text {Off }}$ and $\Delta t_{C}^{\text {Off }}$ represent the time for getting off trains from A, B and C. $\Delta t_{A}^{O n}, \Delta t_{B}^{O n}$ or $\Delta t_{C}^{O n}$ denotes the time for getting on a connecting train to $\mathrm{A}, \mathrm{B}$ or $\mathrm{C}$. The $4^{\text {th }}$ and $5^{\text {th }}$ rows of these tables have the minimum and maximum headways and tracking intervals of the trains arriving at $\mathrm{X}$ from $\mathrm{A}, \mathrm{B}$, and $\mathrm{C}$ and the connecting trains departing from $\mathrm{X}$ to $\mathrm{A}, \mathrm{B}$ and $\mathrm{C} . C_{A B}$ or $C_{A C}$ indicates the number of necessary trains which depart from $\mathrm{X}$ to $\mathrm{B}$ or $\mathrm{C}$ and are before departures boarded at $\mathrm{X}$ by all the transfer passengers who get off one arrived train from A and make the same transfer. Similarly, $C_{B A}$ or $C_{C A}$ is the number of trains departing from $\mathrm{X}$ to $\mathrm{A}$ and boarded at $\mathrm{X}$ by all the transfer passengers getting off one arrived train from $\mathrm{B}$ or $\mathrm{C}$ for the same transfer.

Table I: Parameters of the proposed model for the morning rush hour of Xierqi Station.

\begin{tabular}{|c|c|c|c|c|c|}
\hline$P_{k}$ & $\Delta t_{i j}^{\text {Walk }}$ & $\Delta t_{i}^{O f f}, \Delta t_{j}^{O n}$ & $\begin{array}{c}H_{A r r}^{\text {Max }}, H_{\text {Arr }}^{\text {Min }}, \\
H_{\text {Dep }}^{\text {Max }}, H_{\text {Dep }}^{\text {Min }}\end{array}$ & $\begin{array}{c}h_{\text {Arr }}^{\text {Max }}, h_{\text {Arr }}^{\text {Min }}, \\
h_{\text {Dep }}^{\text {Max }}, h_{\text {Dep }}^{\text {Min }}\end{array}$ & $C_{\text {Dep }}$ \\
\hline$P_{1}=77.00$ & $\Delta t_{A B}^{\text {Walk }}=92.15$ & $\Delta t_{A}^{\text {Off }}=20.00$ & $H_{A X}^{\text {Max }}=465.00$ & $h_{A X}^{\text {Max }}=210.00$ & $C_{A B}=3$ \\
$P_{2}=23.00$ & $\Delta t_{A C}^{\text {Walk }}=104.65$ & $\Delta t_{B}^{O n}=11.00$ & $H_{A X}^{\text {Min }}=315.00$ & $h_{A X}^{\text {Min }}=150.00$ & $C_{A C}=2$ \\
& & $\Delta t_{C}^{O n}=10.00$ & $H_{X B}^{\text {Max }}=195.00$ & $h_{X B}^{\text {Max }}=150.00$ & \\
& & & $H_{X B}^{\text {Min }}=90.00$ & $h_{X B}^{\text {Min }}=90.00$ & \\
& & & $H_{X C}^{\text {Max }}=255.00$ & $h_{X C}^{\text {Max }}=210.00$ & \\
& & $H_{X C}^{\text {Min }}=90.00$ & $h_{X C}^{\text {Min }}=115.00$ & \\
\hline
\end{tabular}

The peak hours of $X$ on the morning of a commuting day last from 7:00 a.m. to 9:00 a.m. when nearly all the transfer passengers in this station make either Transfer-1 or Transfer- 2 . Few passengers in X make Transfer-3 or Transfer-4 in this time period. Reducing the transfer waiting time of Transfer-1 and Transfer-2 is the most important for the efficient trips of passengers and the secure operation of the station in such time. There are 18 trains successively arriving at $X$ from $A$ and respectively 48 and 43 trains departing in proper order from $\mathrm{X}$ to $\mathrm{B}$ and $\mathrm{C}$ in the morning peak period. The average transfer waiting time of a passenger in morning rush hour is first optimized here with the proposed model by adjusting the arrival time of trains from A. Only a limited number of the transfer passengers whose volume is large are able to board a connecting train departing from $\mathrm{X}$ to $\mathrm{B}$ or $\mathrm{C}$ in morning rush hour because its train cars have become very crowded before its arriving at $\mathrm{X}$. Thence, the ratios of the passengers who get off one train arriving $\mathrm{X}$ and board different connecting trains for the same transfer are almost the same in the morning peak time.

From 5:00 p.m. to 7:00 p.m. is another peak time of $X$ in a commuting day. Transfer-3 and Transfer-4 attract almost all the transfer passengers in this station at this time and little importance is attached to Transfer- 1 or Transfer- 2 in this time period. Only decreasing the transfer waiting time of Transfer-3 and Transfer-4 is able to effectively improve the operation security of the station and successfully save the travel time of passengers in the evening rush hour. In these two hours, 16 trains depart in succession from $\mathrm{X}$ to $\mathrm{A}$, and meanwhile, there are 38 and 41 trains arriving successively at $\mathrm{X}$ from $\mathrm{B}$ and $\mathrm{C}$ respectively. The average transfer waiting time of a passenger in the evening peak hour is optimized by rationalizing the time of trains departing from $\mathrm{X}$ to $\mathrm{A}$ in this study. In the evening rush hour, the transfer passengers getting of trains from $\mathrm{B}$ and $\mathrm{C}$ are always mixed as a whole to make Transfer-3 and Transfer- 
4 at the same time. The total volume of these passengers is very big and usually together assigned equally into different connecting trains to A.

Table II: Parameters of the proposed model for the evening peak period of Xierqi Station.

\begin{tabular}{|c|c|c|c|c|c|}
\hline$P_{k}$ & $\Delta t_{i j}^{\text {Walk }}$ & $\Delta t_{i}^{\text {Off }}, \Delta t_{j}^{O n}$ & $\begin{array}{c}H_{\text {Arr }}^{\text {Max }}, H_{\text {Arr }}^{\text {Min }}, \\
H_{\text {Dep }}^{\text {Max }}, H_{\text {Dep }}^{\text {Min }}\end{array}$ & $\begin{array}{c}h_{\text {Arr }}^{\text {Max }}, h_{\text {Arr }}^{\text {Min }}, \\
h_{\text {Dep }}^{\text {Max }}, h_{\text {Dep }}^{\text {Min }}\end{array}$ & \multicolumn{1}{|c|}{$C_{\text {Dep }}$} \\
\hline$P_{3}=79.00$ & $\Delta t_{B A}^{\text {Walk }}=77.61$ & $\Delta t_{B}^{\text {Off }}=7.00$ & $H_{B X}^{\text {Max }}=300.00$ & $h_{B X}^{\text {Max }}=225.00$ & $C_{B A}=2$ \\
$P_{4}=21.00$ & $\Delta t_{C A}^{\text {Walk }}=66.84$ & $\Delta t_{C}^{\text {Off }}=5.00$ & $H_{B X}^{\text {Min }}=135.00$ & $h_{B X}^{\text {Min }}=100.00$ & $C_{C A}=2$ \\
& & $\Delta t_{A}^{\text {On }}=15.00$ & $H_{C X}^{\text {Max }}=225.00$ & $h_{C X}^{\text {Max }}=150.00$ & \\
& & & $H_{C X}^{\text {Min }}=150.00$ & $h_{C X}^{\text {Min }}=90.00$ & \\
& & & $H_{X A}^{\text {Max }}=630.00$ & $h_{X A}^{\text {Max }}=330.00$ & \\
& & & $H_{X A}^{\text {Min }}=375.00$ & $h_{X A}^{\text {Min }}=150.00$ & \\
\hline
\end{tabular}

Table III: Parameters of the proposed model for the non-peak hours of Xierqi Station.

\begin{tabular}{|c|c|c|c|c|c|}
\hline$P_{k}$ & $\Delta t_{i j}^{\text {Walk }}$ & $\Delta t_{i}^{O f f}, \Delta t_{j}^{O n}$ & $\begin{array}{l}H_{A r r}^{M a x}, H_{A r r}^{M i n}, \\
H_{D e p}^{M a x}, H_{D e p}^{M i n}\end{array}$ & $\begin{array}{l}h_{A r r}^{M a x}, h_{A r r}^{M i n}, \\
h_{D e p}^{M a x}, h_{D e p}^{M i n}\end{array}$ & $C_{D e p}$ \\
\hline$P_{1}=40.00$ & $\Delta t_{A B}^{\text {Walk }}=76.60$ & $\Delta t_{A}^{O f f}=13.00$ & $H_{A X}^{M a x}=885.00$ & $h_{A X}^{M a x}=630.00$ & $C_{A B}=1$ \\
\hline$P_{2}=13.00$ & $\Delta t_{A C}^{\text {Walk }}=84.49$ & $\Delta t_{B}^{O n}=5.00$ & $H_{A X}^{M i n}=660.00$ & $h_{A X}^{M i n}=450.00$ & $C_{A C}=1$ \\
\hline$P_{3}=32.00$ & $\Delta t_{B A}^{\text {Walk }}=68.58$ & $\Delta t_{C}^{O n}=5.00$ & $H_{X B}^{M a x}=540.00$ & $h_{X B}^{M a x}=480.00$ & $C_{B A}=1$ \\
\hline$P_{4}=15.00$ & $\Delta t_{C A}^{\text {Walk }}=57.58$ & $\Delta t_{B}^{O f f}=5.00$ & $H_{X B}^{M i n}=420.00$ & $h_{X B}^{M i n}=330.00$ & $C_{C A}=1$ \\
\hline & & $\Delta t_{C}^{O f f}=5.00$ & $H_{X C}^{M a x}=510.00$ & $h_{X C}^{M a x}=465.00$ & \\
\hline & & $\Delta t_{A}^{O n}=13.00$ & $H_{X C}^{M i n}=345.00$ & $h_{X C}^{M i n}=270.00$ & \\
\hline & & & $H_{B X}^{M a x}=510.00$ & $h_{B X}^{M a x}=456.00$ & \\
\hline & & & $H_{B X}^{M i n}=450.00$ & $h_{B X}^{M i n}=270.00$ & \\
\hline & & & $H_{C X}^{M a x}=555.00$ & $h_{C X}^{M a x}=480.00$ & \\
\hline & & & $H_{C X}^{M i n}=420.00$ & $h_{C X}^{M i n}=330.00$ & \\
\hline & & & $H_{X A}^{M a x}=930.00$ & $h_{X A}^{M a x}=630.00$ & \\
\hline & & & $H_{X 4}^{\operatorname{Min}}=675.00$ & $h_{X A}^{M i n}=450.00$ & \\
\hline
\end{tabular}

Representative non-peak hours of $X$ in a commuting day are from 1:00 p.m. to 3:00 p.m. when volumes of the passengers making all the four transfers in this station are comparatively small and their ratios are relatively similar to each other. Though the transfer passenger flows in such time is not enough to do harm to the operation safety of $\mathrm{X}$, comparatively long time consumed by waiting for connecting trains still causes much inconvenience to the transfer passengers. In this time period, the numbers of the trains departing in proper order from $\mathrm{X}$ to $A$ and arriving at $X$ from $A$ in succession are both 10. At the same time, there are 17 trains which come successively to $\mathrm{X}$ from $\mathrm{B}$ and then depart from $\mathrm{X}$ towards $\mathrm{C}$ and there are another 17 trains coming from $\mathrm{C}$ stop at this station in succession and continue their transports to B. In this work, the average transfer waiting time of a passenger in the off-peak hours is minimized by optimizing the time of trains arriving at and departing from $\mathrm{X}$ on Changping Line. 
According to the comparative simulations of the passenger transfers connected with trains which have different arrival and departure time before and after the optimizations, the average waiting time saving results owing to the above-explained optimizations of the arriving and departing time of trains for respectively the morning and evening peak hours and the off-peak period of $\mathrm{X}$ in a commuting day are computed with:

$$
\Delta T \%=\frac{T^{\text {Original }}-T^{\text {Optimized }}}{T^{\text {Original }}} \times 100 \%
$$

where $\Delta T \%$ represents the decrease percentage of $T$ after rationalizing the arrival and departure time of trains, unit: \%; $T^{\text {Original }}$ is the $T$ of the transfers studied in simulation based on the original arrival and departure time of trains, unit: s; and $T^{\text {optimized }}$ donates the $T$ of the transfers simulated according to the optimized arrival and departure time of trains, unit: $s$. Detailed optimization effect is presented in Table IV. It is proved that not only the average waiting time of all the transfers but also the waiting time of each transfer in X has been truly decreased for every time period. This indicates that the transfer efficiencies of all the transfer passengers in this station are improved indeed. Meanwhile, the transfer operation of $\mathrm{X}$ has become much safer from both integral and fractional viewpoints.

Table IV: Effect of optimizing the average transfer waiting time in Xierqi Station.

\begin{tabular}{|c|c|c|c|c|}
\hline \multicolumn{2}{|c|}{ Time Periods } & 7:00 a.m. - 9:00 a.m. & 1:00 p.m. - 3:00 p.m. & 5:00 p.m. - 7:00 p.m. \\
\hline \multirow{3}{*}{$\begin{array}{c}\text { All } \\
\text { Transfers }\end{array}$} & $T^{\text {Original }}$ & 219.47 & 376.86 & 408.39 \\
\hline & $T^{\text {Optimized }}$ & 201.52 & 312.56 & 384.09 \\
\hline & $\Delta T \%$ & 8.18 & 17.06 & 6.33 \\
\hline \multirow{3}{*}{ Transfer-1 } & $T^{\text {Original }}$ & 236.67 & 180.00 & - \\
\hline & $T^{\text {Optimized }}$ & 215.59 & 130.50 & - \\
\hline & $\Delta T \%$ & 8.91 & 27.50 & - \\
\hline \multirow{3}{*}{ Transfer-2 } & $T^{\text {Original }}$ & 161.91 & 220.50 & - \\
\hline & $T^{\text {Optimized }}$ & 154.41 & 172.50 & - \\
\hline & $\Delta T \%$ & 4.63 & 21.77 & - \\
\hline \multirow{3}{*}{ Transfer-3 } & $T^{\text {Original }}$ & - & 546.00 & 393.31 \\
\hline & $T^{\text {Optimized }}$ & - & 466.50 & 373.90 \\
\hline & $\Delta T \%$ & - & 14.56 & 5.19 \\
\hline \multirow{3}{*}{ Transfer-4 } & $T^{\text {Original }}$ & - & 676.50 & 465.14 \\
\hline & $T^{\text {Optimized }}$ & - & 591.00 & 422.43 \\
\hline & $\Delta T \%$ & - & 12.64 & 10.11 \\
\hline
\end{tabular}

\section{CONCLUSIONS}

Now it is confirmed that the newly developed optimization model is able to effectively reduce the waiting time of each transfer in an urban rail station from an integrated transfer operation perspective by adjusting the arrival and departure time of trains in view of the transfer passenger flows and the capacity of their feeder services. Accordingly, not only the travel time of a passenger making a transfer in a rail station can be truly decreased but also the operation of the station will synchronously become more efficient with faster evacuations of its transfer passengers. This is significant for ensuring the operation security of an urban rail station in particular for its rush hours as well as providing transfer convenience to the trip of a passenger.

Of course, improvements to this study are still necessary on several aspects. For instance, delays of trains have not been considered in current research. As a result, a measure capable 
of rationally setting buffer time on train diagrams could be implemented in future work. In addition, the computation efficiency of the proposed algorithm for the new model is sometimes not satisfying enough at this stage and therefore need to be substantially increased in the future.

\section{ACKNOWLEDGEMENTS}

This study is supported by the Fundamental Research Funds for the Central Universities (2014JBM062).

\section{REFERENCES}

[1] Liu, S.; Gong, D. (2014). Modelling and simulation on recycling of electric vehicle batteries using agent approach, International Journal of Simulation Modelling, Vol. 13, No. 1, 79-92, doi:10.2507/IJSIMM13(1)CO1

[2] Feng, X. (2011). Optimization of target speeds of high-speed railway trains for traction energy saving and transport efficiency improvement, Energy Policy, Vol. 39, No. 12, 7658-7665, doi:10.1016/j.enpol.2011.08.051

[3] Research Group of the Annual Report of China Urban Mass Transit (2013). 2012 Annual Report of China Urban Mass Transit, Beijing Jiaotong University Press, Beijing

[4] Kaspi, M.; Raviv, T. (2013). Service-oriented line planning and timetabling for passenger trains, Transportation Science, Vol. 47, No. 3, 295-311, doi:10.1287/trsc.1120.0424

[5] Ceder, A.; Golany, B.; Tal, O. (2001). Creating bus timetables with maximal synchronization, Transportation Research Part A: Policy and Practice, Vol. 35, No. 10, 913-928, doi:10.1016/S0965-8564(00)00032-X

[6] Sato, K.; Tamura, K.; Tomii, N. (2013). A MIP-based timetable rescheduling formulation and algorithm minimizing further inconvenience to passengers, Journal of Rail Transport Planning \& Management, Vol. 3, No. 3, 38-53, doi:10.1016/j.jrtpm.2013.10.007

[7] Vansteenwegen, P.; Van Oudheusden, D. (2006). Developing railway timetables which guarantee a better service, European Journal of Operational Research, Vol. 173, No. 1, 337-350, doi:10.1016/j.ejor.2004.12.013

[8] Edelkamp, S; Schroedl, S. (2012). Heuristic Search: Theory and Applications, Morgan Kaufmann, Waltham

[9] Wong, R. C.; Yuen, T. W.; Fung, K. W.; Leung, J. M. (2008). Optimizing timetable synchronization for rail mass transit, Transportation Science, Vol. 42, No. 1, 57-69, doi:10.1287/trsc. 1070.0200

[10] Liebchen, C. (2008). The first optimized railway timetable in practice, Transportation Science, Vol. 42, No. 4, 420-435, doi:10.1287/trsc. 1080.0240

[11] Diestel, R. (2010). Graph Theory (4 ${ }^{\text {th }}$ Edition), Springer-Verlag, Heidelberg

[12] Khani, A.; Shafahi, Y. (2011). Transfer optimization in transit networks: Headway and departure time coordination, $201114^{\text {th }}$ International IEEE Conference on Intelligent Transportation Systems, Washington DC, 1531-1536, doi:10.1109/ITSC.2011.6082846

[13] Hsu, S. C. (2010). Determinants of passenger transfer waiting time at multi-modal connecting stations, Transportation Research Part E: Logistics and Transportation Review, Vol. 46, No. 3, 404-413, doi:10.1016/j.tre.2009.12.002

[14] Ying, Z.; Jun, L.; Hui, Z. (2013). Transit transfer optimization with capacity constraint consideration: Evidence from Chengdu bus, 2013 International Conference on Management Science and Engineering, Harbin, 2023-2028, doi:10.1109/ICMSE.2013.6586542

[15] Ponnuswamy, S. (2012). Railway Transportation: Engineering, Operation and Management, Alpha Science Int., Oxford

[16] Vuchic, V. R. (2005). Urban Transit: Operations, Planning and Economics, John Wiley \& Sons, Hoboken 